\title{
Remarks on the Burgers Equation
}

\author{
M. ROSENBLATt \\ University of California, San Diego, California
}

(Received 21 November 1967)

\begin{abstract}
Periodic and aperiodic solutions of the Burgers equation $u_{t}+u u_{x}=\mu u_{x z}, \mu>0$, are studied in this paper. A harmonic analysis of the solutions is carried out and the form of the spectrum is estimated for large time. Corresponding estimates of energy decay are also made. In Burgers' work on this equation, the case in which $\mu \downarrow 0$ with $t$ fixed, and one then lets $t \rightarrow \infty$, is studied. In our investigation, a fixed value of $\mu>0$ is taken and then one lets $t \rightarrow \infty$. A similar analysis is also carried out for an irrotational solution of a similar 3-dimensional system of equations. For large time and moderate wavenumbers there is, to the first order, a drift of spectral mass from low wavenumbers to higher wavenumbers. Comments are also made on the asymptotic distribution of a class of random solutions.
\end{abstract}

\section{INTRODUCTION}

Periodic and aperiodic solutions of the Burgers equation are studied in this paper. A harmonic analysis of the solutions is carried out and the form of the spectrum is estimated asymptotically for large time. Corresponding estimates of energy decay are also made. A similar analysis is also carried out for an irrotational solution of a similar 3-dimensional system of equations. For large time and moderate wavenumbers there is, to the first order, a drift of spectral mass from low wavenumbers to higher wavenumbers. Comments are also made on the asymptotic distribution of a class of random solutions of the equations.

\section{THE BURGERS EQUATION}

Burgers introduced the equation

$$
u_{t}+u u_{x}=\mu u_{x x}, \quad \mu>0,
$$

as a model equation for $t>0,-\infty<x<\infty$, in order to get some insight into turbulence. ${ }^{1} \mathrm{Hopf}^{2}$ and $\mathrm{Cole}^{3}$ 'showed that the study of this equation could be reduced to that of the heat equation. Specifically, if $u_{0}(x)=u(0, x)$ is continuous and

as $|x| \rightarrow \infty$, then

$$
\int_{0}^{x} u_{0}(\xi) d x=o\left(x^{2}\right)
$$

$$
u(x, t)=\frac{\int_{-\infty}^{\infty} \frac{x-y}{t} \exp \left[-\frac{1}{2 \mu} F(x, y, t)\right] d y}{\int_{-\infty}^{\infty} \exp \left[-\frac{1}{2 \mu} F(x, y, t)\right] d y,}
$$

with

$$
F(x, y, t)=\frac{(x-y)^{2}}{2 t}+\int_{0}^{y} u_{0}(y) d y,
$$

1 G. K. Batchelor, The Theory of Homogeneous Turbulence (Cambridge University Press, 1953).

E. Hopf, Commun. Pure Appl. Math. 3, 201 (1950).

3 J. D. Cole, Quart. Appl. Math. 9, 226 (1951). is the unique solution of the initial-value problem. Letting

$$
\begin{aligned}
\varphi_{0}(y) & =\exp \left[-\frac{1}{2 \mu} \int_{0}^{\nu} u_{0}(\eta) d \eta\right], \\
\varphi(x, t) & =(4 \pi \mu t)^{-\frac{1}{2}} \int_{-\infty}^{\infty} \varphi_{0}(y) \exp \left[-\frac{(x-y)^{2}}{4 \mu t}\right] d y,
\end{aligned}
$$

it is clear that $\varphi(x, t)$ is a nonnegative solution of the heat equation

and that

$$
\varphi_{t}=\mu \varphi_{x x}
$$

$$
u(x, t)=-2 \mu \frac{\partial}{\partial x} \log \varphi(x, t) .
$$

Because of the continuity of $u_{0}$,

$$
\lim _{\substack{t \rightarrow 0 \\ y \rightarrow x}} u(t, y)=u_{0}(x)
$$

It is sometimes convenient to consider solutions of Burgers equation on the circle (or equivalently periodic solutions of the equation). One then has a corresponding result on existence and uniqueness of the solution without condition (1.2). A standard argument indicates that the solutions of (1.1), of period $2 \pi$ with continuous initial data $u_{0}(x)$ and

$$
\int_{0}^{2 \pi} u_{0}(x) d x=0,
$$

are given in terms of solutions $\varphi(x, t)$ of the heat equation with period $2 \pi$,

$$
\varphi(x, t)=\sum y_{j} \exp \left(-j^{2} \mu t\right) \exp (i j x),
$$

with initial condition

$$
\varphi(x, 0)=\varphi_{0}(x)=\sum y_{j} \exp (i j x)
$$

given by (1.4) in terms of $u_{0}(x)$. Again, $\varphi$ is positive so that $y_{j}=\bar{y}_{-j}$ and $y_{0}>0$. Then

$$
u(x, t)=-2 \mu \frac{\partial}{\partial x} \log \varphi(x, t) .
$$


It will sometimes be convenient to look at random solutions of the Burgers equation that are stationary in $x$. We look in particular at some stationary solutions obtained from solutions of the heat equation that are stationary in $x$. If $\varphi$ is a solution of the heat equation on the circle $[0,2 \pi)$ (or, equivalently, a periodic solution with period $2 \pi$ ) it takes the form (1.9). Stationarity up to second-order moments implies that the coefficients $y_{j}$ in representation (1.10) are orthogonal

$$
E y_{j} \bar{y}_{k}=\delta_{j-k} a_{j} \geq 0, \quad \sum a_{j}<\infty .
$$

Stationarity up to second-order moments for solutions of the heat equation on the full real line implies that one can write

$$
\varphi(x, t)=\int_{-\infty}^{\infty} e^{i x \lambda} e^{-\mu \lambda^{2} t} d Y(\lambda),
$$

where $Y(\lambda)$ is a process with orthogonal increments

$$
E d Y(\lambda) d \bar{Y}(\mu)=\delta_{\lambda-\mu} d F(\lambda),
$$

with $F$ a bounded nondecreasing function. The sequence $\left\{a_{j}\right\}$ and function $F(\lambda)$ correspond to the second-order spectral decomposition of the process in the periodic and general case.

\section{PERIODIC SOLUTIONS}

Consider a periodic solution of the Burgers equation with period $2 \pi$ and spatial mean zero; that is, condition (1.8) is satisfied. For continuous initial data, it has already been noted that the solution can be given in terms of a positive solution $\varphi(x, t)$ of the heat equation (1.5) with $y_{0}>0$. The continuity of initial data implies that

$$
\sum_{i}\left|y_{j}\right|^{2}<\infty .
$$

In view of (1.6), the solution $u(x, t)$ of the Burgers equation can be written,

$$
\begin{aligned}
u(x, t) & =-2 \mu \frac{\sum_{j} y_{j}(i j) \exp \left(-j^{2} \mu t\right) \exp (i j x)}{\sum_{j} y_{j} \exp \left(-j^{2} \mu t\right) \exp (i j x)} \\
& =\sum_{k} Z_{k}(t) \exp (i k x) .
\end{aligned}
$$

If we formally look at the Burgers equation in the Fourier domain,

$$
Z_{k}^{\prime}(t)+\sum_{j} Z_{k-j}(t) Z_{j}(t) i j=-\mu k^{2} Z_{k}(t)
$$

is obtained for the Fourier coefficients $Z_{k}(t)$ of $u(x, t)$. This equation is certainly satisfied for sufficiently large $t$. We shall obtain estimates of $Z_{k}(t)$ with error term valid for large $t$ and moderate $k$ from the representation (2.2). These estimates will be used to show that, in the range of large $t$ and moderate $k$, there is a unidirectional transfer of spectral mass from wavenumbers $1,2, \cdots, k$ to $k+1$ to the first order. This is consistent with some of the heuristic arguments employed in the discussion of nonlinear phenomena like turbulence (see Refs. 1-3).

Make the strong assumption

$$
\sum_{j \neq 0}\left|y_{j}\right|<y_{0},
$$

or the stronger assumption (for a later computation)

$$
\sum_{j \neq 0}\left|y_{j}\right| j^{2}<y_{0},
$$

so that the expansion

$$
u(x, t)=2 \mu \frac{\psi_{x}}{y_{0}-\psi}=2 \mu \sum_{j=0}^{\infty} y_{0}^{-j-1} \frac{\partial}{\partial x} \psi^{j+1} /(j+1)
$$

is valid, where

$$
\varphi(x, t)=y_{0}-\psi .
$$

Even if (2.4) or $\left(2.4^{\prime}\right)$ are not satisfied, they will be satisfied at some later time $t$ by $y_{j} \exp \left(-j^{2} \mu t\right)$ so that the qualitative features of our conclusions are valid for any periodic solution satisfying (1.8).

Now

$$
\begin{aligned}
Z_{k}(t)=2 \mu i k & \sum_{j=0}^{\infty} \frac{1}{j+1} \sum_{s_{1}+\cdots \underbrace{}_{\substack{s_{i} \neq 0 \\
s_{j+1}=k}} y_{s_{1}} \cdots y_{s_{j+1}}} \\
& \times \exp \left[-\left(s_{1}^{2}+\cdots+s_{j+1}^{2}\right) \mu t\right] y_{0}^{-j-1}
\end{aligned}
$$

and

$$
\begin{aligned}
Z_{k}^{\prime}(t)= & 2 \mu^{2} i k \sum_{j=0}^{\infty} \frac{1}{(j+1)} \\
& \times \sum_{s_{1}+\cdots} \sum_{\substack{s_{i} \neq 0 \\
s_{j+1}-k}} y_{s_{1}} \cdots y_{s_{j+1}}\left(-s_{1}^{2}-\cdots-s_{j+1}^{2}\right) \\
& \times \exp \left[-\left(s_{1}^{2}+\cdots+s_{j+1}^{2}\right) \mu t\right] y_{0}^{-j-1} .
\end{aligned}
$$

Our aim is now to give the principal terms for $Z_{k}(t)$, $Z_{k}^{\prime}(t)$ together with bounds for the error terms. First consider $Z_{k}(t)$ with $k \geq 1\left[Z_{-k}(t)=Z_{k}(t)\right.$ since the solutions are real]. The principal term for large $t$ is

$$
2 \mu i\left(y_{1} / y_{0}\right)^{k} \exp (-k \mu t)
$$

corresponding to the term in the inner summation of (2.7) with $j+1=k$ and $s_{1}=s_{2}=\cdots=s_{k}=1$. All the other terms of the inner summation corresponding 
to $j+1=k$ are in total bounded by

$$
\begin{aligned}
& 2 \mu k\left[\sum_{s \neq 0}\left|y_{s} / y_{0}\right| \exp \left(-s^{2} \mu t\right)\right]^{k-1} \\
& \times\left[\sum_{s \neq 0,1}\left|y_{s}\right| y_{0} \mid \exp \left(-s^{2} \mu t\right)\right] .
\end{aligned}
$$

The total of the terms of the inner summation corresponding to fixed $j+1$ with $j+1>k$ is bounded by

$$
2 \mu k\left[\sum_{s \neq 0}\left|y_{s} / y_{0}\right| \exp \left(-s^{2} \mu t\right)\right]^{j+1}
$$

Under assumption (2.4), the total of the terms of the inner summation corresponding to fixed $j+1$ with $j+1<k$ is bounded by

$$
\text { where } \begin{gathered}
2 \mu k \exp \left\{-M_{j+1} \mu t\right\}, \\
M_{j+1}=\min _{\substack{s_{1}+\cdots+s_{j+1}=k \\
s_{i} \neq 0}}\left\{s_{1}^{2}+\cdots+s_{j+1}^{2}\right\} .
\end{gathered}
$$

However, $M_{j+1} \geq k+2\{k-(j+1)\}$ if $j+1<k$. Thus, for large $t$,

$$
\left|Z_{k}(t)-2 \mu i\left(y_{1} / y_{0}\right)^{k} e^{-k \mu t}\right|<\mu k C \exp [-(k+1) \mu t],
$$

where $C$ is an absolute constant. Under assumption $\left(2.4^{\prime}\right)$ a similar argument implies that $Z_{k}^{\prime}(t)$ is to the first order

$$
\text { and that }-2 \mu^{2} i k\left(y_{1} / y_{0}\right)^{k} e^{-k \mu t}
$$

$\left|Z_{k}^{\prime}(t)+2 \mu^{2} i k\left(y_{1} / y_{0}\right) e^{-k \mu t}\right|<\mu^{2} k C \exp [-(k+1) \mu t]$,

with $C$ an absolute constant. Let us now look at Eq. (2.3) with the estimates given by (2.14) and (2.16). Notice that

$$
\left|\sum_{\substack{j<00 \\ \text { or } j>k}} Z_{k-j}(t) Z_{j}(t) i j\right| \leq C k^{2} e^{-\mu t} e^{-(k+1) \mu t},
$$

where $C$ is an absolute constant. This implies that

$$
\begin{aligned}
Z^{\prime}(t)+\sum_{j=1}^{k-1} Z_{k-j}(t) Z_{j}(t) i j+\mu k^{2} Z_{k}(t) \\
=O\left\{\left(k^{3} e^{-\mu t}\right) \exp [-(k+1) \mu t]\right\},
\end{aligned}
$$

as $t \rightarrow \infty$ where the constant, corresponding to the $O$ order of magnitude estimate, is independent of $k$. At the very least, if $k^{3} \exp (-\mu t)=o(1)$ as $t \rightarrow \infty$, the error estimate on the right of Eq. (2.18) is negligible compared to the individual terms on the left of the equation since each of those terms is precisely of order of magnitude $\exp (-k \mu t)$. It follows that we have a drift of spectral mass from lower wavenumbers
$1 \leq j<k$ to $k$ through the convolution term

$$
\sum_{j=1}^{k-1} Z_{k-j}(t) Z_{j}(t) i j,
$$

as given in a number of heuristic discussions of turbulence. This is enough to counterbalance the dissipative term $-\mu k^{2} Z_{k}(t)$ to such an extent that

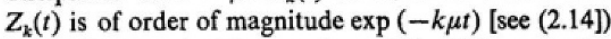
rather than $\exp \left(-k^{2} \mu t\right)$, as it would be if the convolution transfer term did not enter. Notice that all three terms of Eq. (2.3) are of order $\exp (-k \mu t)$.

$\mathrm{Cole}^{4}$ has recently obtained estimates related to (2.14).

\section{ENERGY ESTIMATES}

We first consider a real deterministic solution of the Burgers equation on the real line. Let $u$ be a solution with $u_{t}, u_{x}$ bounded and $u \in L$,

$$
\lim _{|x| \rightarrow \infty} u(x)=0 .
$$

The assumptions on $u$ imply that $u \in L^{2}$. On multiplying the Burgers equation by $u$ and integrating with respect to $x$, the following is obtained:

$$
\begin{aligned}
\int_{-N}^{N}\left[u u_{t}+u^{2} u_{x}\right] d x \\
=\frac{1}{2} \frac{d}{d t} \int_{-N}^{N} u^{2} d x+\frac{1}{3}\left[u^{3}(N)-u^{3}(-N)\right] \\
=\mu \int_{-N}^{N} u u_{x x} d x \\
=\mu u u_{x}||_{-N}^{N}-\mu \int_{-N}^{N} u_{x}^{2} d x
\end{aligned}
$$

The relation

$$
\frac{1}{2} \frac{d}{d t} \int u^{2} d x=-\mu \int u_{x}^{2} d x
$$

is obtained in the limit as $N \rightarrow \infty$ and describes the rate of decay of energy.

A similar argument can be used to give a corresponding result in the case of a random solution of the Burgers equation stationary in $x$. It will be more than enough to assume that moments of $u$ and its partial derivatives $u_{t}, u_{x}$, and $u_{x x}$ exist up to fourth order. Multiply the Burgers equation by $u$ and take expectations to obtain

$$
E\left\{u u_{t}+u_{x} u^{2}\right\}=\mu E\left(u u_{x z}\right) .
$$

The simple equalities

$$
E u_{x} u^{2}=\frac{1}{3} \frac{\partial}{\partial x} E u^{3}=0
$$

J. D. Cole (unpublished). 
and

$$
\frac{\partial}{\partial x} E u u_{x}=E u_{x}^{2}+E u u_{x x}=0
$$

imply that

$$
\frac{1}{2} \frac{d}{d t} E u^{2}=-\mu E u_{x}^{2} .
$$

We shall get estimates of the energy and the rate of decay of energy when $t$ is large for a class of solutions of the Burgers equation in both the deterministic solution of the Burgers equation of the form $u=$ $-2 \mu \varphi_{x}(c+\varphi)^{-1}$, where $\max |\varphi|<c$ and

$$
\varphi(x, t)=\int \exp \left(i x \lambda-\mu t \lambda^{2}\right) h(\lambda) d \lambda,
$$

where $h \in L$ and is bounded. Now

$$
\int|u(x, t)|^{2} d x=4 \mu^{2} \int\left|\frac{\varphi_{x}}{c+\varphi}\right|^{2} d x
$$

and, since $|\varphi| \rightarrow 0$ as $t \rightarrow \infty$,

$$
\int|u(x, t)|^{2} d x \cong 4 \mu^{2} c^{-2} \int\left|\varphi_{x}\right|^{2} d x,
$$

as $t \rightarrow \infty$. The energy content of the solution $u$ of the Burgers equation given above is the same as that of the solution $2 \mu c^{-1} \varphi_{x}$ of the heat equation as $t \rightarrow \infty$. The rate of decay of total energy is governed by $\int\left|u_{x}\right|^{2}$ $d x$ and

$$
u_{x}(x, t)=-2 \mu\left[(c+\varphi) \varphi_{x x}-\varphi_{x}^{2}\right](c+\varphi)^{-2} .
$$

Thus

$$
\int\left|u_{x}(x, t)\right|^{2} d x \cong 4 \mu^{2} c^{-4} \int\left|(c+\varphi) \varphi_{x x}-\varphi_{x}^{2}\right|^{2} d x
$$

as $t \rightarrow \infty$. Since

$$
\int\left|\varphi_{x x}\right|^{2}|c+\varphi|^{2} d x \cong c^{2} \int\left|\varphi_{x x}\right|^{2} d x
$$

as $t \rightarrow \infty$ and

$$
\int(c+\varphi) \varphi_{x x} \varphi_{x}^{2} d x=\int \varphi \varphi_{x x} \varphi_{x}^{2} d x=-\frac{1}{3} \int \varphi_{x}^{4} d x,
$$

it follows that

$$
\int\left|u_{x}\right|^{2} d x \cong 4 \mu^{2} c^{-2} \int\left|\varphi_{x x}\right|^{2} d x+\frac{20}{3} \mu^{2} c^{-4} \int\left|\varphi_{x}\right|^{4} d x
$$

as $t \rightarrow \infty$. Since

$$
\begin{aligned}
\sup _{x}\left|\varphi_{x}(x, t)\right| & \leq \int \exp \left(-\mu t \lambda^{2}\right)|\lambda||h(\lambda)| d \lambda \\
& =O\left[(\mu t)^{-1}\right]
\end{aligned}
$$

as $t \rightarrow \infty$, then

$\int\left|\varphi_{x}(t, x)\right|^{4} d x \leq C(\mu t)^{-2} \int \lambda^{2} \exp \left(-2 \mu t \lambda^{2}\right)|h(\lambda)|^{2} d \lambda$ as $t \rightarrow \infty$ with $C$ a constant. If $h(\lambda) \cong k \lambda^{\alpha}, \alpha \geq 0$, and $k \neq 0$, as $\lambda \downarrow 0$, we shall have the second term on the right of relation (3.9) of smaller order of magnitude than the first as $t \rightarrow \infty$, so that

$$
\int\left|u_{x}\right|^{2} d x \cong 4 \mu^{2} c^{-2} \int\left|\varphi_{x x}\right|^{2} d x
$$

as $t \rightarrow \infty$. The asymptotic rate of decay of energy will then be the same as for the solution $2 \mu c^{-1} \varphi_{x}$ of the heat equation. Notice that our discussion of total energy and its rate of decay for large $t$ depends on the low-frequency range of $\lambda$ in the neighborhood of zero.

Similar conclusions can be obtained for a random solution $u=-2 \mu \varphi_{x}(c+\varphi)^{-1}$, where

$$
\varphi(x, t)=\int \exp \left(i x \lambda-\mu t \lambda^{2}\right) d Z(\lambda),
$$

with $\varphi(x, 0)$ a bounded process stationary in $x$, of absolute value less than $c$ and mean zero. Here $Z(\cdot)$ is the random spectral function corresponding to $\varphi(x, 0)$. We shall assume that $\varphi(x, 0)$ has second-order spectral density $f(\lambda)$ and fourth-order cumulant spectral density $g\left(\lambda_{1}, \lambda_{2}, \lambda_{3}\right)$. These will be well defined and continuous if the second-order moments and fourth-order cumulants are integrable as functions of the one and three linearly independent differences of spatial arguments respectively (see Ref. 5 for a discussion of higher-order spectra). As before, $|\varphi| \rightarrow 0$ as $t \rightarrow \infty$, so that

$$
E u^{2} \cong 4 \mu^{2} c^{-2} E \varphi_{x}^{2}=4 \mu^{2} c^{-2} \int \lambda^{2} \exp \left(-2 \mu t \lambda^{2}\right) f(\lambda) d \lambda
$$

as $t \rightarrow \infty$. The rate of dissipation of energy is given by (3.5), with $u_{x}$ given by (3.8). Then

with

$$
E u_{x}^{2} \cong 4 \mu^{2} c^{-4} E\left|(c+\varphi) \varphi_{x x}-\varphi_{x}^{2}\right|^{2},
$$

$$
E\left|(c+\varphi) \varphi_{x x}-\varphi_{x}^{2}\right|^{2} \cong c^{2} E \varphi_{x x}^{2}+\frac{5}{3} E \varphi_{x}^{4} .
$$

Now, $E \varphi_{x}^{4}=c_{4}\left(\varphi_{x}\right)+3 \sigma^{4}\left(\varphi_{x}\right)$, where $c_{4}$ is the fourth cumulant of $\varphi_{x}$ and $\sigma^{2}\left(\varphi_{x}\right)$ is the variance of $\varphi_{x}$. Thus

$$
\begin{aligned}
E \varphi_{x}^{4}= & 3\left[\int \lambda^{2} \exp \left(-2 \mu t \lambda^{2}\right) f(\lambda) d \lambda\right]^{2} \\
& +\int \lambda_{1} \lambda_{2} \lambda_{3}\left(-\lambda_{1}-\lambda_{2}-\lambda_{3}\right) \\
& \times \exp \left\{-\mu t\left[\lambda_{1}^{2}+\lambda_{2}^{2}+\lambda_{3}^{2}+\left(\lambda_{1}+\lambda_{2}+\lambda_{3}\right)^{2}\right]\right\} \\
& \times g\left(\lambda_{1}, \lambda_{2}, \lambda_{3}\right) d \lambda_{1} d \lambda_{2} d \lambda_{3} .
\end{aligned}
$$

${ }^{5}$ Ya. G. Sinai, Theory Probability Appl. (USSR) 8, 429 (1963). 
Assuming that $f$ and $g$ are bounded and that $f$ is not zero in the neighborhood of zero,

while

$$
E \varphi_{x x}^{2} \sim(\mu t)^{-\frac{1}{2}},
$$

$$
E \varphi_{x}^{4}=O\left[(\mu t)^{-3}\right],
$$

as $t \rightarrow \infty$. In that case the principal contribution to the decay of energy would be expected from the term $E \varphi_{x x}^{2}$ when $t$ is large.

\section{A SOLUTION ON THE REAL LINE}

In this section we shall again look at a solution of the Burgers equation of the form $u=2 \mu \Psi_{x}(c-\Psi)^{-1}$ with $\max |\Psi|<c$ and $\Psi$ given by (3.6). The function $h$ is assumed to be bounded, real, and continuous with $h(0)=c>0$. Our object is to get an estimate of the Fourier transform $f(\lambda, t)$ (with respect to $x$ ) of $u(x, t)$ at a fixed $\lambda \neq 0$ as $t \rightarrow \infty$. This is a limited parallel to the computation carried out in Sec. 2 for a periodic solution. Notice that as $t$ gets large this will fall outside of both the energy-containing and energydissipative range for which $\lambda \sim(\mu t)^{-\frac{1}{2}}$ as $t \rightarrow \infty$ by arguments like those given in the section on energy estimates.

Now

$$
u(x, t)=2 \mu \sum_{k=0}^{\infty} \frac{y_{0}^{-k-1} \frac{\partial}{\partial x} \Psi^{k+1}}{(k+1)} .
$$

Consider the Fourier transform of $\Psi^{k}(k>1)$

$$
\begin{aligned}
(2 \pi)^{-(k-1)} & \int \exp \left\{-\mu t\left[\left(\lambda-\sum_{1}^{k-1} \lambda_{i}\right)^{2} t+\sum_{1}^{k-1} \lambda_{i}^{2}\right]\right\} \\
& \times h\left[\lambda-\sum_{1}^{k-1} \lambda_{i}\right] \prod_{1}^{k-1} h\left(\lambda_{i}\right) d \lambda_{1} \cdots d \lambda_{k-1} .
\end{aligned}
$$

Set

$$
\lambda_{i}=\frac{\lambda}{k}+(2 \mu t)^{-\frac{1}{2}} z_{i} .
$$

Integral (4.2) can then be rewritten as

$$
\begin{aligned}
& (2 \pi)^{-(k-1)} \exp \left(-\mu t \lambda^{2} / k\right)(2 \mu t)^{-(k-1) / 2} \\
& \times \int \exp \left\{-\frac{1}{2}\left[\left(\sum_{1}^{k-1} z_{i}\right)^{2}+\sum_{1}^{k-1} z_{i}^{2}\right]\right\} h\left[\frac{\lambda}{k}-(2 \mu t)^{-\frac{1}{2}} \sum_{1}^{k-1} z_{i}\right] \\
& \times \prod_{1}^{k-1} h\left(\frac{\lambda}{k}+(2 \mu t)^{-\frac{1}{2}} z_{i}\right) d z_{1} \cdots d z_{k-1} .
\end{aligned}
$$

This suggests the estimate

$$
\exp \left(-\mu t \lambda^{2} / k\right)\left\{h\left(\frac{\lambda}{k}\right)\right\}^{k}(4 \pi \mu t)^{-(k-1) / 2} k^{-\frac{1}{2}}
$$

for integral (4.3) to the first order and therefore

$$
2 \mu i \lambda \sum_{k=1}^{\infty} \exp \left(-\mu t \lambda^{2} / k\right)\left[y_{0}^{-1} h\left(\frac{\lambda}{k}\right)\right]^{k}(4 \pi \mu t)^{-(k-1) / 2} k^{-\frac{3}{2}}
$$

as an estimate of $f(\lambda, t)$. For $\lambda \sim(\mu t)^{-1}$, it is clear that only the term corresponding to $k=1$ is of interest as $t \rightarrow \infty$. Consider a fixed $\lambda>0$ and assume that

$$
h(\lambda)=h_{0}+\lambda h_{1}+o(\lambda), \lambda>0,
$$

in the neighborhood of $\lambda=0$. The following approximation is obtained by taking into account those terms in the summation (4.5) for values of $k$ in the neighborhood of $k_{\max }$, which maximizes the typical term

$$
\begin{array}{r}
f(\lambda, t) \sim 2 \mu i \lambda \exp \left(-\lambda\left\{\mu t\left[\frac{1}{2} \log \left(\frac{4 \pi \mu t y_{0}^{2}}{h_{0}^{2}}\right)\right]\right\}^{\frac{1}{2}}\right. \\
+O[\log (\mu t)]) .
\end{array}
$$

\section{AN OVERSIMPLIFIED VERSION OF THE NAVIER-STOKES EQUATIONS}

A three-dimensional example like the Burgers equation can be obtained by looking at the NavierStokes system of equations without the continuity equation and the pressure terms, and by looking for solutions that are gradients. We wish to consider Cauchy's problem for the equations

$$
\frac{\partial \mathbf{v}}{\partial t}+(\mathbf{v} \cdot \nabla) \mathbf{v}=\mu \Delta \mathbf{v}, \quad \mu>0,
$$

where $\mathbf{v}=\left(v_{1}, v_{2}, v_{3}\right)$ is a 3-vector depending on 3 spatial variables and time, and the initial vector $\mathbf{v}_{0}=\mathbf{v}(x, 0)$ satisfies curl $\mathbf{v}_{0}=0$. This system is briefly mentioned at the end of Cole's paper ${ }^{3}$ but is studied at some length in the paper of Kuznetsov and Rozhdestvenskii. ${ }^{6}$ The fact that curl $\mathbf{v}_{0}=0$ implies that a solution with this initial specification is irrotational for all time. By introducing a scalar function $\varphi$ satisfying

$$
\mathbf{v}=-2 \mu \boldsymbol{\nabla} \log \varphi,
$$

the initial-value problem for Eq. (5.1) is reduced to that of

$$
\frac{\partial \varphi}{\partial t}=\mu \Delta \varphi .
$$

If we set $\varphi(x, t)=y_{0}-\Psi$, with $\max |\varphi|<y_{0}$ as before,

$$
-2 \mu \log \varphi-2 \mu \log y_{0}=2 \mu \sum_{j=0}^{\infty} y_{0}^{-j-1} \Psi^{n+1} /(j+1) .
$$

N. N. Kuznetsov and B. L. Rozhdestvenskii, Z. Vyeisl. Mat. Fiz. 1, 217 (1961). 
An argument like that given in Sec. 4 now suggests approximating the Fourier transform of $\Psi^{k}(k \geq 1)$ by

if $\Psi$ is given by

$$
\exp \left(-\mu t \lambda^{2} / k\right)\left\{h\left(\frac{\lambda}{k}\right)\right\}^{k}(4 \pi \mu t)^{-\frac{2}{2}(k-1)} k^{-\frac{2}{2}}
$$

$$
\Psi(\mathbf{x}, t)=\int \exp \left(i \mathbf{x} \cdot \boldsymbol{\lambda}-\mu t \lambda^{2}\right) h(\lambda) d \boldsymbol{\lambda}
$$

where $\mathbf{x}$ and $\lambda$ are 3 -vectors, and $h$ is bounded, real, and continuous. One can then proceed further and obtain estimates somewhat like (4.7) under further assumptions on $h$ analogous to those made in Sec. 4 . Instead of proceeding in this direction, we shall consider periodic solutions of system (5.1) in the manner of Sec. 2.

Let $\mathbf{v}$ be an irrotational solution of Eq. (5.1) with period $2 \pi$ in each spatial variable and

$$
\begin{aligned}
\int_{0}^{2 \pi} v^{(1)}(\mathbf{x}, t) d x_{1} & =\int_{0}^{2 \pi} v^{(2)}(\mathbf{x}, t) d x_{2} \\
& =\int_{0}^{2 \pi} v^{(3)}(\mathbf{x}, t) d x_{3}=0 .
\end{aligned}
$$

Then the solution $\mathbf{v}$ is given by (5.2) in terms of a solution

$$
\varphi=y_{0}-\Psi=y_{0}-\sum_{\mathbf{k} \neq 0} y_{\mathbf{k}} \exp \left(-\mathbf{k}^{2} \mu t\right) \exp (i \mathbf{k} \cdot \mathbf{x})
$$

of the heat equation. Notice that $\mathbf{k}$ is a wavenumber 3-vector of integers and $\mathbf{0}$ is the zero 3 -vector. Assume, as in Sec. 2, that

$$
\sum_{\mathbf{k}}\left|y_{\mathbf{k}}\right| \mathbf{k}^{2}<y_{0},
$$

We can then get estimates for the vectors $\mathbf{z}_{\mathbf{k}}(t)$ and $\mathbf{z}_{\mathbf{k}}^{\prime}(t)$, for large $t$, where the $\mathbf{z}_{\mathbf{k}}(t)$ are the Fourier coefficients in the harmonic analysis of $\mathbf{v}$ :

Then

$$
\mathbf{v}(\mathbf{x}, t)=\sum \mathbf{z}_{\mathbf{k}}(t) e^{i \mathbf{k} \cdot \mathbf{x}}
$$

$$
\begin{aligned}
& \mathbf{z}_{\mathbf{k}}(t)=2 \mu i \mathbf{k} \sum_{j=0}^{\infty} \frac{1}{(j+1)} \\
& \times \sum_{s_{1}+\cdots \substack{\begin{subarray}{c}{* \\
s_{i} \neq 0} }} \\
{s_{j+1}=k}\end{subarray}} e^{-\left(s_{1}{ }^{2}+\cdots+s_{j+1}{ }^{2}\right) \mu t} y_{s_{1}} \cdots y_{s_{j+1}} y_{0}^{-j-1} .
\end{aligned}
$$

For large $t$, it is clear that the first-order contribution to $z_{k}(t)$ in $(5.11)$ will come from vectors $\mathbf{s} \neq 0$ with the following properties. The component $s_{i}$ of $\mathbf{s}$ will be zero if $k_{i}=0$, while $s_{i}=0,1\left(s_{i}=0,-1\right)$ if $k_{i}>0$ $\left(k_{i}<0\right)$. Call this set of vectors $U(\mathbf{k})$. Thus, if all the components of $\mathbf{k}$ are positive, $U(\mathbf{k})$ consists of the vectors $(1,0,0),(0,1,0),(0,0,1),(1,1,0),(1,0,1)$, $(0,1,1)$, and $(1,1,1)$. If $\mathbf{k}$ has its first component negative and the others zero, $U(\mathbf{k})$ contains only
$(-1,0,0)$. Let us set

$$
A(\mathbf{k})=\sum_{j \geq 0} \frac{1}{j+1} \sum_{\substack{\left.s_{1}+\cdots+s_{j+1}=\mathbf{k} \\ s_{i} \in U / \mathbf{k}\right)}} y_{\mathbf{s}_{1}} \cdots y_{\mathbf{s}_{j+1}} y_{0}^{-j-1} .
$$

Then, for fixed $\mathbf{k}$,

$$
\mathbf{z}_{\mathbf{k}}(t)=2 \mu i \mathbf{k} A(\mathbf{k}) \exp \left[-\sum_{i=1}^{3}\left|k_{i}\right| \mu t\right]\left[1+o\left(e^{-\mu t}\right)\right]
$$

and

$$
\begin{aligned}
\mathbf{z}_{\mathbf{k}}^{\prime}(t)=-2 \mu^{2} i \mathbf{k} A(\mathbf{k})\left(\sum_{i=1}^{3}\left|k_{i}\right|\right) \\
\quad \times \exp \left(-\sum_{i=1}^{3}\left|k_{i}\right| \mu t\right)\left[1+o\left(e^{-\mu t}\right)\right],
\end{aligned}
$$

as $t \rightarrow \infty$. Notice that the analog of the system (2.3) is

$$
\mathbf{z}_{\mathbf{k}}^{\prime}(t)+\sum_{\mathbf{k}^{\prime}} i\left[\mathbf{k}^{\prime} \cdot \mathbf{z}_{\mathbf{k}-\mathbf{k}^{\prime}}(t)\right] \mathbf{z}_{\mathbf{k}^{\prime}}(t)=-\mu|\mathbf{k}|^{2} \mathbf{z}_{\mathbf{k}}(t) .
$$

This is certainly satisfied by (5.11), for $t$ sufficiently large. We shall just briefly remark on the analog of Eq. (2.18) for wavevectors $k$ all of whose components are nonnegative. Given any two wavevectors $\mathbf{k}$ and $\mathbf{k}^{\prime}, \mathbf{k} \geq \mathbf{k}^{\prime}$ means that $\mathbf{k}-\mathbf{k}^{\prime}$ is a vector all of whose components are nonnegative. The estimates (5.14) and (5.15) with error terms imply that

$$
\begin{array}{r}
\mathbf{z}_{\mathbf{k}}^{\prime}(t)+\sum_{\mathbf{k} \geq \mathbf{k}^{\prime} \geq 0} i\left[\mathbf{k}^{\prime} \cdot \mathbf{z}_{\mathbf{k}-\mathbf{k}^{\prime}}(t)\right] \mathbf{z}_{\mathbf{k}^{\prime}}(t)+\mu|\mathbf{k}|^{2} \mathbf{z}_{\mathbf{k}}(t) \\
=O\left\{\exp \left[-\left(1+\sum_{i=1}^{3}\left|k_{i}\right|\right) \mu t\right]\right\},
\end{array}
$$

if $\mathbf{k} \geq \mathbf{0}, \mathbf{k} \neq \mathbf{0}$ with $\mathbf{k}$ fixed as $t \rightarrow \infty$. The error estimate is small compared to each of the terms on the left of (5.16) which are of order of magnitude $\exp \left\{-\sum_{i=1}^{3}\left|k_{i}\right| \mu t\right\}$. One has a drift of spectral mass from wavevectors $\mathbf{k}^{\prime}, 0 \leq \mathbf{k}^{\prime} \leq \mathbf{k}$ to $\mathbf{k}$ through the truncated convolution term as before. If $\mathbf{k} \leq 0$, the convolution term would be summed over $\mathbf{k}^{\prime}, \mathbf{k} \leq$ $\mathbf{k}^{\prime} \leq 0$, with a similar modification for $\mathbf{k}$ belonging to some other octant.

\section{ASYMPTOTIC DISTRIBUTION OF A CLASS OF RANDOM SOLUTIONS.}

Let us now consider random solutions of the Burgers equation that are stationary in $x$, Our object is to get some information on the asymptotic distribution of the solution as $t \rightarrow \infty$. The case of periodic solutions satisfying condition (1.8) is rather uninteresting, since it is clear from (2.2) that

$$
u(x, t) \cong-2 \mu\left(y_{1} / y_{0}\right) e^{i x} e^{-\mu t}
$$

as $t \rightarrow \infty$. The asymptotic distribution of $u(x, t)$ (assuming that $y_{0}>\delta>0$ with probability one) is determined by the joint distribution of $y_{0}$ and $y_{1}$. 
Consider now a random solution of the Burgers equation given by

$$
u=2 \mu \Psi_{x}\left(c-\Psi^{-1},\right.
$$

with $c$ a positive constant and $\Psi$ a strictly stationary solution of the heat equation (1.5) in $x$ with

$$
\max \left|\Psi_{0}(x)\right|<c, \quad E \Psi_{0}(x) \equiv 0,
$$

where $\Psi_{0}(x)=\Psi(x, 0)$. Notice that (6.3) implies that $\max |\Psi(x, t)|\left\langle c\right.$ for all $t>0$. The stationarity of $\Psi_{0}$ in $x$ implies that $u_{0}(x)=u(x, 0), u(x, t)$, and $\Psi(x, t)$ are strictly stationary. Let the second-order spectral distribution function of $\Psi_{0}$ be $F(\lambda)$,

$$
E \Psi_{0}(y+x) \Psi_{0}(y)=\int e^{i x \lambda} d F(\lambda) .
$$

Since $u_{0}(x)=2 \mu \Psi_{0 x}\left(c-\Psi_{0 x}\right)^{-1}$, it is clear that at the very least we ought to have

$$
\int \lambda^{2} d F(\lambda)<\infty
$$

in order that $u_{0}(x)$ be well defined at least in mean square. Let us assume that $\Psi_{0}$ is strongly (or uniformly) mixing in the following sense. ${ }^{7.8}$ Let $A, B$ be two events with $A$ determined by conditions on the random variables $\Psi_{0}(x)$ with $x \leq y$ and $B$ determined by the random variables $\Psi_{0}(x)$ with $x \geq y+d, d>0$. The process $\Psi_{0}$ is said to be strongly mixing if

$$
|P(A B)-P(A) P(B)|<\alpha(d),
$$

where $\alpha(d)$ is a function of $d$ that tends to zero as $d \rightarrow \infty$ and that otherwise is independent of $A, B$, and $y$. This condition of strong mixing is a strong form of asymptotic independence as the events $A$ and $B$ are determined by random variables that are further and further removed spatially. Conditions sufficient for strong mixing are discussed in Refs. 7 and 8 . Notice that strong mixing for $\Psi_{0}$ implies that $u_{0}$ is strongly mixing. Since

$$
\Psi(x, t)=\int_{-\infty}^{\infty}(4 \pi \mu t)^{-\frac{1}{2}} \exp \left\{-\frac{(x-y)^{2}}{4 \mu t}\right\} \Psi_{0}(y) d y,
$$

it is clear that $\max |\Psi(x, t)| \rightarrow 0$ as $t \rightarrow \infty$ and that

as $t \rightarrow \infty$. Now:

$$
u(x, t) \cong 2 \mu c^{-1} \Psi_{x},
$$

$\Psi_{x}(x, t)=-\int(4 \pi \mu t)^{-\frac{t}{2}} \exp \left\{-\frac{(x-y)^{2}}{4 \mu t}\right\} \Psi_{0 y}(y) d y$.

Equation (6.8) indicates that $\Psi_{x}$ is obtained from $\Psi_{0 x}$ by narrow band pass filtering about frequency zero and one knows that $\Psi_{0 x}$ is strongly mixing (since $\Psi_{0}$

\footnotetext{
"A. N. Kolmogorov and Y. A. Rozanov, Theory Probability
Appl. (USSR) 5, 204 (1960).

M. Rosenblatt, Quart. Appl. Math. 18, 387 (1961).
}

is strongly mixing). Under appropriate conditions on second and fourth order moments (see Ref. 8), one would expect $\Psi_{x}$ to be asymptotically normally distributed (when properly normalized) as $t \rightarrow \infty$. The assumption that $\Psi_{0}$ is strongly mixing implies that $F$ is absolutely continuous with spectral density $f(\lambda)$. But then $\Psi_{0 x}$ has spectral density $\lambda^{2} f(\lambda)$. One of the conditions required for asymptotic normality of $\Psi_{x}$ is that the variance of $(4 \pi \mu t)^{\frac{1}{2}} \Psi_{x}$ diverge as $t \rightarrow \infty$. But this variance

$$
\sigma^{2}\left[(4 \pi \mu t)^{\frac{1}{2}} \Psi_{x}\right]=4 \pi \mu t \int \lambda^{2} e^{-2 \mu t \lambda^{2}} f(\lambda) d \lambda=O(1) .
$$

We do not have divergence of (6.9) as $t \rightarrow \infty$ and so $u(x, t)$ as given by $(6.7)$, is typically not asymptotically normal. The following objection might be brought against our argument. Even though $u$ as given by (1.6) is stationary in $x$, the function $\varphi$ might not be and it is this class of random solutions one ought to look at. Nonetheless, we shall see that random solutions of (5.1) stationary in $\mathbf{x}$ that are obtained from corresponding stationary solutions of the heat equation (5.3) by (5.2) are typically asymptotically normal under analogous conditions. This could be thought of as a difference between the one- and three-dimensional equations.

Let us consider a random solution of Eq. (5.1) given by

$$
\mathbf{v}=2 \mu(c-\Psi)^{-1} \nabla \Psi
$$

with $c$ a positive constant and $\Psi$ a strictly stationary solution of (5.1) in $\mathbf{x}$ with

$$
\max _{\mathbf{x}}\left|\Psi_{0}(\mathbf{x})\right|<c, \quad E \Psi_{0}(\mathbf{x}) \equiv 0,
$$

where $\Psi_{0}(\mathbf{x})=\Psi(\mathbf{x}, 0)$. Let $F$ be the second-order spectral distribution function of $\Psi_{0}$

$$
E \Psi_{0}(\mathbf{y}+\mathbf{x}) \Psi_{0}(\mathbf{y})=\int \exp (i \mathbf{x} \cdot \lambda) d F(\lambda) .
$$

One would have to require that

$$
\int|\lambda|^{2} d F(\lambda)<\infty,
$$

if $\mathbf{v}$ is to be well defined in mean square. The following definition is a possible generalization of the concept of strong mixing for a process $\Psi_{0}(\mathbf{x})$ with parameter $\mathbf{x}$ a point in 3-space. Consider any two events $A, B$ determined by the random variables $\left\{\Psi_{0}(\mathbf{x}), \mathbf{x} \in I\right\}$ and $\left\{\Psi_{0}\left(\mathbf{x}^{\prime}\right), \mathbf{x}^{\prime} \in I^{\prime}\right\}$, respectively. Let $C_{1}, C_{2}$ be the convex hulls of the set of points $\{\mathbf{x}, \mathbf{x} \in I\}$ and $\left\{\mathbf{x}^{\prime}, \mathbf{x}^{\prime} \in I^{\prime}\right\}$, respectively. Set $d$ equal to the distance between $C_{1}, C_{2}$. We shall say that $\Psi_{0}(\mathbf{x})$ is strongly mixing if condition (6.6) is satisfied for all events $A$, $B$, where $\alpha(d)$ is a function of $d$ that tends to zero as $d \rightarrow \infty$. If $\Psi_{0}$ is strongly mixing, then $F$ is absolutely 
continuous with spectral density $f(\lambda)$. As $t \rightarrow \infty$ one as $t \rightarrow \infty$ if $f(\lambda)$ is continuous and bounded away can show that from zero at $\lambda=0$. Actually, less stringent conditions

and

$$
\mathbf{v}(\mathbf{x}, t) \cong 2 \mu c^{-1} \nabla \Psi
$$

$(4 \pi \mu t)^{\frac{3}{2}} \nabla \Psi(\mathbf{x}, t)=-\int_{-\infty}^{\infty} \exp \left\{-\frac{|\mathbf{x}-\mathbf{y}|^{2}}{4 \mu t}\right\} \nabla \Psi_{0}(\mathbf{y}) d \mathbf{y}$.

The spectral density of $(4 \pi \mu t)^{\frac{3}{2}} \Psi_{x_{i}}(\mathbf{x}, t)$ is

$\sigma^{2}\left[(4 \pi \mu t)^{\frac{3}{2}} \Psi_{x_{i}}(\mathbf{x}, t)\right]$

$$
=(4 \pi \mu t)^{3} \int \lambda_{i}^{2} \exp \left(-2 \mu t|\lambda|^{2}\right) f(\lambda) d \lambda \rightarrow \infty,
$$

on $f(\lambda)$ at zero would suffice for (6.16). Again, it is clear from (6.15) that we are narrow band pass filtering about $\lambda=0$. But now because of (6.16), broad conditions on fourth-order moments assure asymptotic normality of (6.10).

\section{ACKNOWLEDGMENTS}

I should like to thank Professor Burgers, Professor Saffman, and Professor Whitham for their helpful comments. 